\title{
Properties of the Social Discount Rate and Intertemporal Negative Externality in the Utility or Production Function
}

\author{
Masayuki Otaki \\ Institute of Social Sciences, The University of Tokyo, Tokyo, Japan \\ Email: ohtaki@iss.u-tokyo.ac.jp
}

Received 20 February 2016; accepted 14 March 2016; published 17 March 2016

Copyright (C) 2016 by author and Scientific Research Publishing Inc.

This work is licensed under the Creative Commons Attribution International License (CC BY). http://creativecommons.org/licenses/by/4.0/

\begin{abstract}
This study examines the role of the social discount rate, as applied to the efficient allocation of carbon dioxide $\left(\mathrm{CO}_{2}\right)$ emissions, which provokes serious intertemporal negative externalities such as global warming. Two approaches exist in introducing the intertemporal negative externality brought about by such emissions into economic theory. One is the utility-function approach, and the other is the production-function approach. The former is well-suited to the case in which climate change directly threatens the living standard of human, for example, through a drastic degradation of the environment. The latter approach becomes relevant to case in which global warming is expected to lead to productivity stagnation particular to the additional costs of adaptation.
\end{abstract}

\section{Keywords}

Utility Function with a Negative Externality, Production Function with a Negative Externality, Social Discount Rate, Efficiency in Emissions Allocation

\section{Introduction}

Global warming can be regarded as a type of intertemporal congestion problem, which originates from the abuse of our non-renewable atmosphere. Excess emissions of $\mathrm{CO}_{2}$, provoked by unlimited combustion of fossil fuels are a crucial factor in global warming. Theoretically speaking, this can be seen as an application of the club goods theory as follows. The deterioration in the quality of the atmosphere due to excess emissions decreases the service that the atmosphere can provide us. For each individual emitter the $\mathrm{CO}_{2}$ contribution is negligible, and congestion is as entirely external for them. However, as the foregoing discussion suggests, this congestion phenomenon is internal for the overall global economy. It is particularly serious and complicated to resolve ow- 
ing to its dynamic nature. With continued emissions, the concentration of $\mathrm{CO}_{2}$ in the atmosphere gradually increases over time, leading to more severe global warming. Accordingly, determining methods of evaluating future damage become vital, making the issue of the social discount rate highly significant.

In addition, in a market economy, the social discount rate is closely related to carbon tax. This is because the optimal carbon price (tax) should be equalized with the capitalized value of carbon by the social discount rate. One will find how dear the carbon price (tax rate) is and sensitive to the social discount rate.

There is an important distinction over which function is more such seriously affected by this negative externality: the utility function or the production function. One can argue that such distinction is meaningless and that the negative externality affects both functions. Nevertheless, there are two schools of thought in theoretical environmental economics. One is the production function approach. The typical examples are Nordhaus [1] and Kuninori and Otaki [2]. The other is the utility function approach (e.g., Uzawa [3], Otaki [4] [5]). It seems an acute issue to analyze whether the implications concerning the optimal social discount rate are dependent on the approach chosen.

This study is an attempt to clarify the following two theoretical issues. One is to explore the property of optimal social discount rate if it exists. The other is to consider whether such properties are affected by the choice of the foregoing two models.

The rest of the study is organized as follows: Section 2 constructs an optimal growth model in which the intertemporal negative externality originating from excess $\mathrm{CO}_{2}$ emissions affects the utility function. The properties of optimal social discount rate and carbon tax are also considered. Section 3 transforms the model with the negative externality in the production function, and compares it with the results obtained in the former section. Section 4 provides some brief concluding remarks.

\section{Utility Function Approach}

Utility function approaches assumes that the intertemporal negative externality originating from excessive $\mathrm{CO}_{2}$ emissions directly affects people's utility. It is assumed that a strictly concave instantaneous utility function, $u_{t}$, is represented by

$$
u(t) \equiv u\left(c_{t},-E_{t}\right) \mathrm{e}^{-\rho t}, u_{1}, u_{2}>0
$$

where $c_{t}, E_{t}$ denote consumption and accumulated emissions, respectively. $\rho$ is the social discount rate applied to emissions. $u_{i}$ is the partial derivative with respect to the $i$-th argument of the function.

It is assumed that emissions are partially absorbed mainly by oceans and forests, and also increases proportionately with consumption. Accordingly, the carbon cycle can be described by the following differential equation. That is,

$$
\dot{E}_{t}=-\alpha E_{t}+\frac{c_{t}}{\beta}
$$

where $\alpha$ is the absorption ratio of $\mathrm{CO}_{2}$, and $\beta$ denotes the efficiency of production measured by the amount of goods produced by per emitted $\mathrm{CO}_{2}$ tonnage.

The maximization problem can be formatted as

$$
\max _{c_{t}} \int_{0}^{+\infty} u(t) \mathrm{d} t \text {, subject to (2). }
$$

The corresponding Hamiltonian, $H_{t}^{U}$, of this problem is defined as

$$
H_{t}^{U} \equiv u\left(c_{t},-E_{t}\right) \mathrm{e}^{-\rho t}+\lambda_{t}^{U}\left[-\alpha\left[-E_{t}\right]-\frac{c_{t}}{\beta}\right]
$$

It should be noted that the state variable, $E_{t}$, is interchanged with $-E_{t}$. The necessary and sufficient conditions of this problem are Equation (2) and

$$
\begin{aligned}
& u_{1}=\frac{\tilde{\lambda}_{t}^{U}}{\beta}, \tilde{\lambda}_{t}^{U} \equiv \lambda_{t}^{U} \mathrm{e}^{\rho t}, \\
& \dot{\tilde{\lambda}}_{t}^{U}=[\alpha+\rho] \tilde{\lambda}_{t}^{U}-u_{2}, \\
& \lim _{t \rightarrow+\infty} \tilde{\lambda}_{t}^{U} E_{t} \mathrm{e}^{-\rho t}=0 .
\end{aligned}
$$


The optimal paths are illustrated by bold arrows in Figure 1. In a situation with excess emissions situation, which corresponds to the lower arrow directing to north-east, optimal consumption is monotonously decreasing and converges to a zero-growth economy unless some technological progress, which absorbs emissions, is developed.

Next, we analyze the properties of the optimal social discount rate in the vicinity of the stationary state. Combining the above two equations in (5) and letting $\dot{\tilde{\lambda}}^{U}=0$, one obtains the following important relationship between optimal consumption and emissions. That is,

$$
\frac{u_{1}}{u_{2}}=\frac{1}{\beta[\alpha+\rho]} .
$$

The left-hand side of Equation (6) corresponds to the tangency of an upward-sloping indifference curve II on $(c, E)$ plane as illustrated by Figure 2. By Equation (2), $E=\frac{c}{\alpha \beta}$ holds as indicated by Line $F F$, which represents the feasible allocation in the stationary equilibrium; thus the stationary state is located at the intersection of curve II and FF.

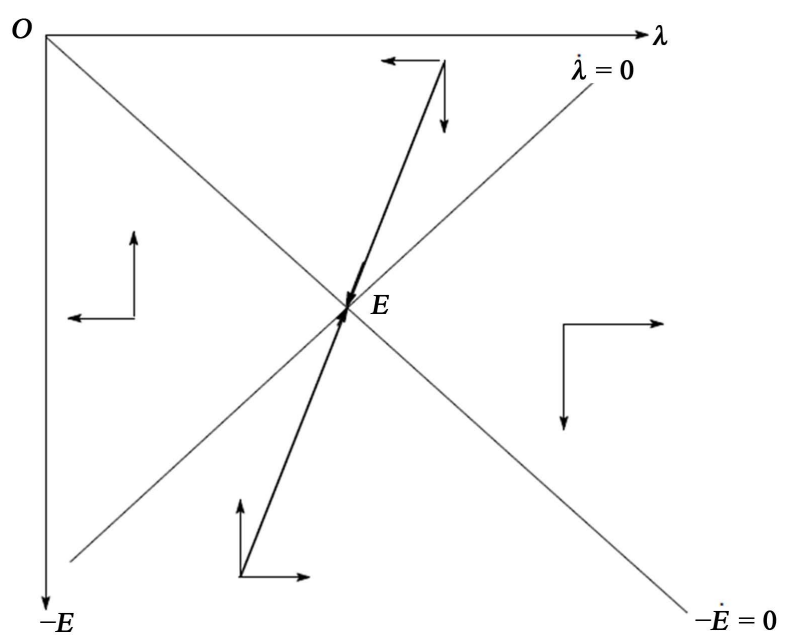

Figure 1. The phase diagram.

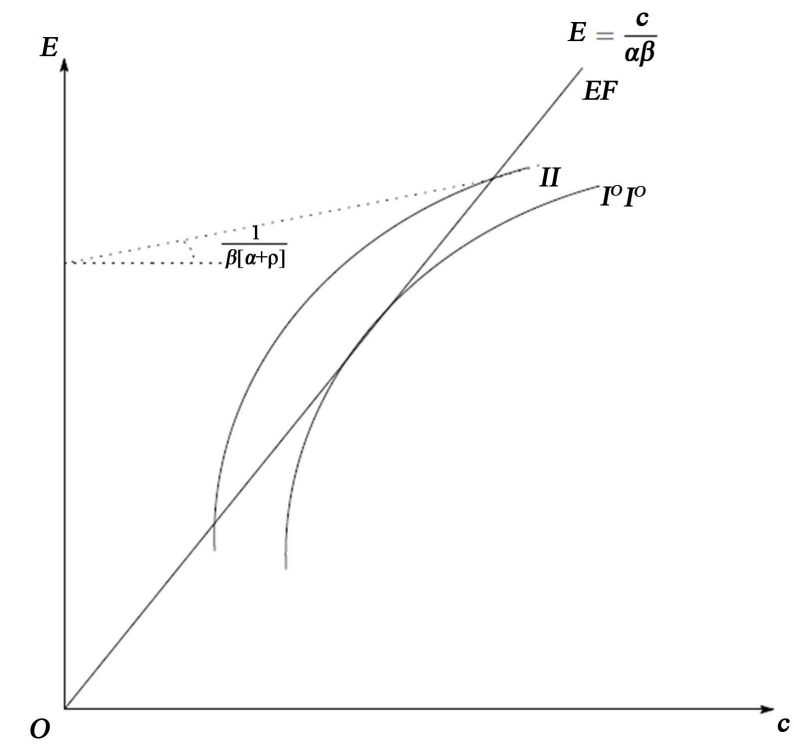

Figure 2. Stationary state and Pareto efficiency. 
Because the indifference curve $I^{0} I^{0}$, which corresponds to zero social discount rate achieves the best allocation, it is evident that nearly-zero discounting (note that the utility integral diverges when zero-discounting is strictly applied) improves economic welfare in the vicinity of the stationary state. To summarize,

\section{Theorem 1}

Welfare is maximized when the social discount rate is preferable as low as possible in the vicinity of stationary state in the utility function approach.

\section{Proof}

At the stationary state in a market economy, a representative individual solves the following static decision problem. That is,

$$
\max _{c, E} u(c,-E) \text {, subject to } E \geq p c,
$$

where $p$ denotes the relative price of consumption goods to the unit saving cost of emissions. Elementary calculus leads us to

$$
\frac{u_{1}}{u_{2}}=p
$$

Combining this equation with Equation (6), one obtains

$$
p=\frac{1}{\beta[\alpha+\rho]}
$$

This completes the proof.

Moreover, additional important information exists in Equation (7). Let a market economy be considered instead of a planned economy. Whenever perfect competition prevails in goods market, the tangency of the indifference curve is equal to the relative price of carbon to consumption goods. Hence, Equation (7) represents the effective carbon price (i.e., tax) in terms of consumption goods. Such a price becomes equal to the capitalized value of goods discounted by the effective social discount rate, $\beta[\alpha+\rho]^{1}$. A society that becomes more tolerant to emissions and more highly discounts future utilities, whilst possibly not, would imply a lower carbon tax in a market economy.

Thus, one obtains

\section{Theorem 2}

A higher social discount rate in a planned economy is equivalent to a lower carbon tax in a market economy.

\section{Proof}

It is clear from Equation (7) that the value of the social discount rate, $\rho$, is in one to one correspond to the carbon price, $p$, to the extent that other parameters are kept invariant. Accordingly, the optimal social planning for some given $\rho$ is equivalent to levying the carbon tax on consumption goods of which value is $p$ in market economy. This discussion completes the proof.

It should be noted that the earth's absorption rate, $\alpha$, is an important factor for determining the carbon price. A large value of $\alpha$ permits a lower carbon price because emitted $\mathrm{CO}_{2}$ does not stay long in the atmosphere. Conversely, if $\alpha$ does not significantly differ from zero, the solution to global warming depends on human beings' ethic, as Otaki [5] proves.

In addition, technological progress is also meaningful in introducing a carbon tax with less friction. Whenever some emissions-saving technology is developed and prevails, this is represented by heightening parameter $\beta$ in the model. As indicated in Equation (7), such a progress lowers the relative price of carbon because the production process produces fewer emissions.

${ }^{1}$ From the equations in (5), the carbon price becomes equal to $\frac{1}{\beta\left[[\alpha+\rho]-\frac{\dot{\tilde{\lambda}}_{t}^{U}}{\tilde{\lambda}_{t}^{U}}\right]}$. Meanwhile it is evident from Figure 1 that $\frac{\dot{\tilde{\lambda}}^{U}}{\tilde{\lambda}^{U}}$ is

strictly positive when an economy is in the situation of excess emissions compared with the stationary state. Accordingly, the optimal carbon price in transition process should be always higher than in the stationary state. In other words, the desired carbon price becomes minimal at the stationary state. 


\section{Production Function Approach}

Under a production function approach, the negative effect of excess emissions is attributed to the production function rather than to the utility function directly. This approach assumes that global warming does not affect the basic living and survival conditions of human beings, but more indirectly, hinders prosperity via lowering productivity. For example, since global warming adjoins climate change, huge costs incurred by adaptation are a typical example.

Let the strictly concave production function, $F$, be denoted as

$$
y_{t}=F\left(k_{t},-E_{t}\right), F_{1}>0, F_{2}>0
$$

where $y_{t}$ is total output of gods, and $k_{t}$ denotes input of goods for production. $\mathrm{CO}_{2}$ emissions originating from anthropogenic combustion of fossil fuels lower productivity; thus, the second argument of $F$ is positive because of the negative externality to production. Then, the differential equation that represents the carbon cycle becomes

$$
\dot{E}_{t}=-\alpha E_{t}+\frac{y_{t}}{\beta}=-\alpha E_{t}+\frac{1}{\beta} F\left(k_{t},-E_{t}\right)
$$

The optimization problem to be solved is

$$
\max _{c_{t}} \int_{0}^{+\infty} v\left(c_{t}\right) \mathrm{e}^{-\rho t} \mathrm{~d} t \text {, subject to Equation (9) }
$$

where $v$ is a strictly concave instantaneous utility function. The corresponding Hamiltonian, $H_{t}^{P}$, is

$$
H_{t}^{P} \equiv v\left(F\left(k_{t},-E_{t}\right)-k_{t}\right) \mathrm{e}^{-\rho t}+\lambda_{t}^{P}\left[-\alpha\left[-E_{t}\right]-\frac{1}{\beta} F\left(k_{t},-E_{t}\right)\right]
$$

The necessary-sufficient conditions are Equation (9) and

$$
\begin{aligned}
& v^{\prime}=\frac{\tilde{\lambda}_{t}^{P}}{\beta} \frac{F_{1}}{\left[F_{1}-1\right]}, \tilde{\lambda}_{t}^{P} \equiv \lambda_{t}^{P} e^{\rho t}, \\
& \dot{\tilde{\lambda}}_{t}^{P}=\left[\alpha+\rho+\frac{F_{2}}{\beta}\right] \tilde{\lambda}_{t}^{P}-v^{\prime} F_{2}, \\
& \lim _{t \rightarrow+\infty} \tilde{\lambda}_{t}^{P} E_{t} \mathrm{e}^{-\rho t}=0 .
\end{aligned}
$$

The dynamics of this economy are described by two differential Equations (9) and (12). Figure 3 is its phase diagram with the optimal paths shown by bold arrows.

By evaluating Equation (9) and the top two equations in (12) at the stationary state, one obtains

$$
\frac{\mathrm{d} E}{\mathrm{~d} c}=\frac{F_{1}-1}{F_{2}}=\frac{1}{\beta[\alpha+\rho]}
$$

The left-hand side of Equation (13) is the slope of an isoquant. Figure 4 illustrates stationary state in production function approach model. Curve $F^{P} F^{P}$ is the feasible set, which corresponds to Equation (9) evaluated at $\dot{\tilde{\lambda}}^{P}=0$. Curves $I Q, I Q^{O}$ are isoquants in $(c, E)$ plane. The same logic as in the case of the utility-function approach can be applied; thus, the relative price of carbon becomes equal to the tangency of isoquant, $\frac{1}{\beta[\alpha+\rho]}$, in the production-function approach. This is because the tangency corresponds to the factor price whenever perfect competition prevails. Accordingly,

\section{Theorem 3}

Regardless of whether one relies on the utility-function or production-function approach, the optimal carbon instationary state is equal to $\frac{1}{\beta[\alpha+\rho]}$. In addition, the relationship between the carbon price and the social discount rate is invariant. 


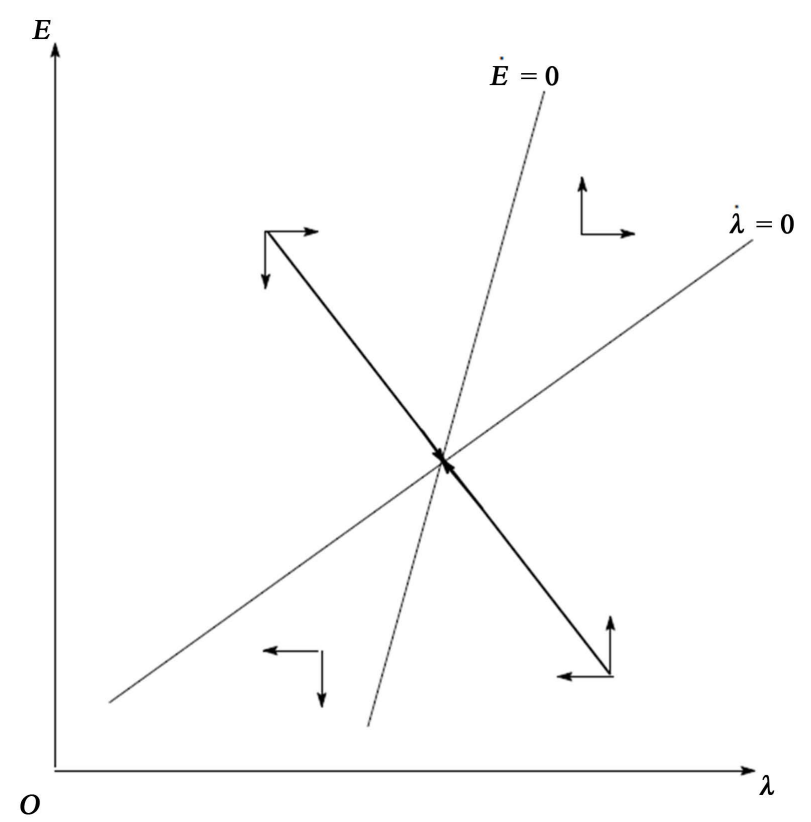

Figure 3. The phase diagram.

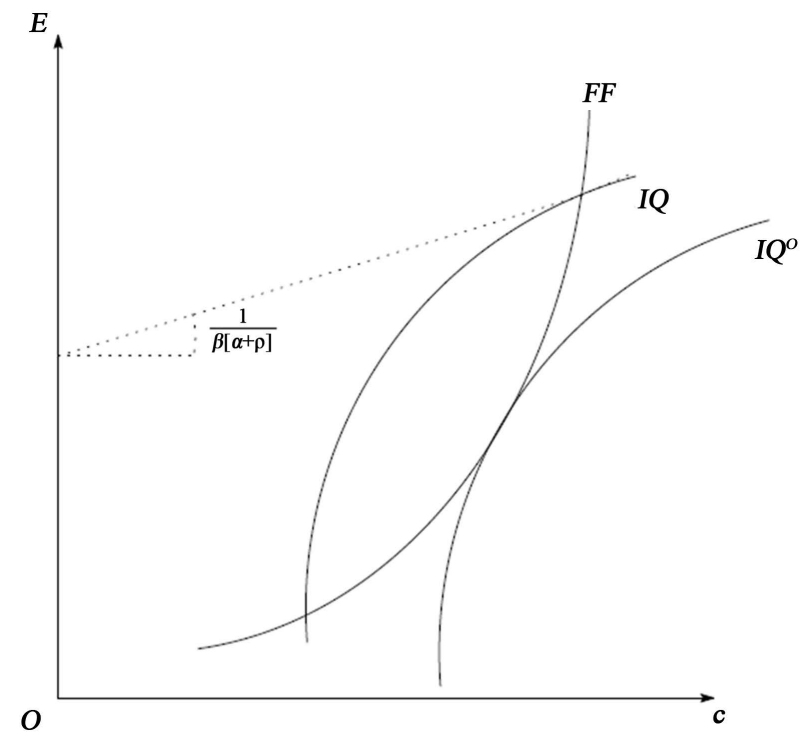

Figure 4. Stationary state and carbon price.

Theorem 3 implies that there is no significant difference in the calculation methods of the carbon price in the vicinity of a stationary equilibrium. In this sense, whether one adopts a utility-function or production-function approach is immaterial.

\section{Concluding Remarks}

This study considered the welfare implications of the social discount rate and calculated the optimal carbon price using an optimal growth model with an intertemporal negative externality. In addition, it examined whether results differ when the negative externality exists in the utility or the production function. The results obtained are as follows:

First, the optimal carbon price in the vicinity of stationary state is independent of the origin of the negative externality: either the utility function or the production function. In addition, the adjustment processes toward a 
stationary state are alike: consumption monotonously decreases and the concentration of $\mathrm{CO}_{2}$ is gradually reduced.

Second, as the social discount rate approaches zero, resource allocation in the vicinity of stationary equilibrium improves. This is because a lower social discount rate evaluates future damages owing to global warming more seriously in a planning economy. Nevertheless, applying a lower discount rate raises the carbon price in a market economy.

Third, the absorption ratio of $\mathrm{CO}_{2}$ by the earth is also a crucial factor in determining the carbon price. If this ratio is high, the carbon price is lower because emitted $\mathrm{CO}_{2}$ does not remain in the atmosphere for a long time. However, it should be noted that the acidification of the oceans may not lead a stable equilibrium in the long run.

Finally, increased carbon-efficiency of production lowers the carbon price. This is because such a technological progress enables an economy to produce more goods in exchange for lower emission. As Kuninori and Otaki [2] shows, it is only when such technological progress continuously accumulates that sustainable growth is feasible.

\section{Acknowledgements}

I am grateful to Professor Morio Kuninori for intensive and incisive discussions. Needless to say, possibly remaining errors are attributed to me. I am also thankful to the subsidy provided by MEXT/JSPS KAKENHI Grant Number 60183761.

\section{References}

[1] Nordhaus, W.D. (2014) Dice-2013R Model of as of November 15, 2013. http://www.econ.yale.edu/ nordhaus/homepage/Web-DICE-2013-April.htm

[2] Kuninori, M. and Otaki, M. (2016) Modified Ramsey Rule, Optimal Carbon Tax and Economic Growth. Atmospheric and Climate Sciences. (In Press)

[3] Uzawa, H. (2009) Economic Theory and Global Warming. Cambridge University Press, Cambridge.

[4] Otaki, M. (2013) The Endogenous Social Discount Rate, Proportional Carbon tax, and Sustainability: Do We Have the Right to Discount Future Generations’ Utility? Environment Systems Research, 2, 1-8. http://dx.doi.org/10.1186/2193-2697-2-1

[5] Otaki, M. (2015) Local Altruism as an Environmental Ethic in $\mathrm{CO}_{2}$ Emissions Control. Atmospheric and Climate Sciences, 5, 433-440. http://dx.doi.org/10.4236/acs.2015.54035 